\title{
An evolutionary algorithm for designing microbial communities via environmental modification
}

\author{
Alan R. Pacheco ${ }^{1}$ and Daniel Segrè*1,2 \\ ${ }^{1}$ Graduate Program in Bioinformatics and Biological Design Center, Boston University, Boston, \\ MA 02215, USA \\ 2 Department of Biology, Department of Biomedical Engineering and Department of Physics, \\ Boston University, Boston, MA 02215, USA \\ * To whom correspondence should be addressed: dsegre@bu.edu
}




\begin{abstract}
Despite a growing understanding of how environmental composition affects microbial community properties, it remains difficult to apply this knowledge to the rational design of synthetic multispecies consortia. This is because natural microbial communities can harbor thousands of different organisms and environmental substrates, making up a vast combinatorial space that precludes exhaustive experimental testing and computational prediction. Here, we present a method based on the combination of machine learning and dynamic flux balance analysis (dFBA) that selects optimal environmental compositions to produce target community phenotypes. In this framework, dFBA is used to model the growth of a community in candidate environments. A genetic algorithm is then used to evaluate the behavior of the community relative to a target phenotype, and subsequently adjust the environment to allow the organisms to more closely approach this target. We apply this iterative process to in silico communities of varying sizes, showing how it can rapidly identify environments that yield desired phenotypes. Moreover, this novel combination of approaches produces testable predictions for the in vivo assembly of microbial communities with specific properties, and can facilitate rational environmental design processes for complex microbiomes.
\end{abstract}

\title{
Keywords
}

Microbial communities, synthetic ecology, genetic algorithm, metabolic modeling. 


\section{Introduction}

Microbial communities are complex ecosystems that are crucial to the health and function of many biomes, from aquatic ecosystems to the human gut [1-5]. In addition to yielding a growing understanding of the composition of various microbial ecosystems [6-8], recent advances in DNA sequencing and synthetic biology have enabled new efforts to engineer synthetic multispecies consortia for a variety of applications [9-11]. For example, multispecies systems have been designed to degrade complex substrates or pollutants [12-15], as well as to produce biofuels and molecules for human consumption [15-18]. Advances such as these portend the advent of new applications in synthetic ecology, in which communities of microbes can be readily designed for a vast number of useful outputs. However, this promise is hampered by the difficulty in genetically manipulating individual organisms at community scales, as well as by the lack of a mechanistic understanding of how environmental factors and interspecies interactions shape communities [1921]. These challenges open up the important question of whether a more accessible parameter, i.e. the chemical composition of the environment, can be modulated to confer specific functions on microbial consortia.

A number of studies have demonstrated the crucial role that changes in environmental composition play in defining microbial community phenotypes, such as in the gut microbiota $[22,23]$ and in aquatic and terrestrial ecosystems $[24,25]$. As natural ecosystems contain complex combinations of different nutrients, studies have also begun to disentangle the nonintuitive relationship between community properties and resource identity and heterogeneity [24,26-29]. These observations point to the manipulation of environmental composition as a promising method for producing synthetic consortia with defined functions. However, these and other recent studies have 
demonstrated that community growth and structure can be so sensitive to environmental composition that even closely-related environments can produce very different communities $[28,30]$. Therefore, in order to reach a phenotype of interest, in practice it often remains necessary to explicitly test a multitude of different specific nutrient combinations - a task that can quickly become experimentally intractable. For example, screening a consortium under all combinations of 20 nutrients - a quantity vastly lower than the number of unique metabolites found in natural settings - would require 1.05 million individual experiments, a scale that remains inaccessible to current conventional microbiological methods. Organism-specific computational models can be deployed to run in silico analogs of these experiments [31-33], though the number of simulations required would also rapidly become computationally intractable for more complex environmental search spaces.

To begin addressing these challenges, we present here the design of a genetic algorithm (GA) framework to rapidly identify environmental compositions that result in target community phenotypes. Our method, conceptually similar to processes used to evolve communities toward specific functions [34-36], searches large spaces of nutrient combinations to produce candidate environmental compositions that optimize a given ecological objective. To test our GA framework, we rely on a large set of in silico community experiments consisting of over 6,000 unique environment-community pairings. While ultra-high-throughput experimental platforms can also generate large sets of community phenotypes in combinatorial environments [37-39], we employ a purely computational technique to demonstrate the ability of our genetic algorithm to identify environments that confer specific target community taxonomic compositions and desired patterns of metabolic secretion and exchange. In addition, we show how this pairing of an evolutionary 
algorithm with models of community ecology allows us to maximize community objectives under a much larger ( 600,000 environments) combinatorial space. In sum, this method is able to reach a desired community design goal without requiring explicit ecological parameters as input, allowing it to serve as a versatile tool for exploration of large combinatorial spaces and future applications in experimental synthetic ecology.

\section{Results}

\section{Generation of microbial community phenotypes in combinatorial environments}

In order to test our search algorithm, we first simulated the growth of multispecies microbial communities under a large number of environmental compositions. This was done via a dynamic flux balance analysis (dFBA) technique [40] using the COMETS software package [41,42], which enables a mechanistic evaluation of community growth and metabolic exchange using experimentally-validated computational models of individual organisms (see Methods). Predictions using dFBA have been shown to recapitulate key microbial phenotypes, while also generating broader statistical mappings of community structure and interactions $[32,33,43,44]$. Moreover, the use of these models allows us to enumerate a complete set of environmentphenotype mappings that is large yet computationally tractable, allowing us to identify every possible community outcome and evaluate the quality of solutions identified by our algorithm against a known optimum. Our mapping was generated by simulating the growth of 13 -species communities in a variety of environmental compositions. The in silico organisms that make up our communities were selected as they represent a diverse cross-section of taxa and metabolic capabilities (see Methods), in theory allowing us to maximize the variability of yields, taxonomic compositions, and interspecies interactions across different environments. We used combinations 
of up to 4 of 20 different carbon sources in order to generate a total of 6,196 unique environmental compositions, to which we added equal amounts of all 13 organisms (see Methods). The growth of these communities in all environments was then simulated with COMETS over a 24-hour timespan.

Our simulated communities displayed high degrees of compositional variability across the environmental conditions we tested (Figure 1a). Specifically, six in silico organisms (B. subtilis, E. coli, P. aeruginosa, S. boydii, S. coelicolor, and S. oneidensis) reached relative abundances of more than $50 \%$ in at least one environment, and all organisms encountered at least one environment in which they could not grow. Organism relative abundances displayed mean variances of 0.02 , mean species richness values of $3.30 \pm 0.99$, and mean Shannon entropy values of $1.29 \pm 0.49$ (Figure 1b, c), which were comparable to those of similarly-sized communities assayed experimentally [28]. Moreover, we encountered a wide distribution in the number of metabolic exchanges across environments. We define a metabolic exchange as the transfer of a unique metabolite from one organism to another, and found that our environments resulted in $435.49 \pm 106.49$ such transfers on average (Figure 1d). Interestingly, the failure of six organisms (K. pneumoniae, L. lactis, P. gingivalis, $R$. sphaeroides, S. cerevisiae, and Z. mobilis), many of which have a variety of metabolic auxotrophies [45-48], to grow in any environment suggests that the levels of metabolic exchange observed in our communities was not enough to sustain these more specialized organisms. In sum, these results recapitulate elements of the often nonintuitive relationship between environmental composition and community structure observed in nature, serving as a suitable dataset on which to test our search algorithm. 

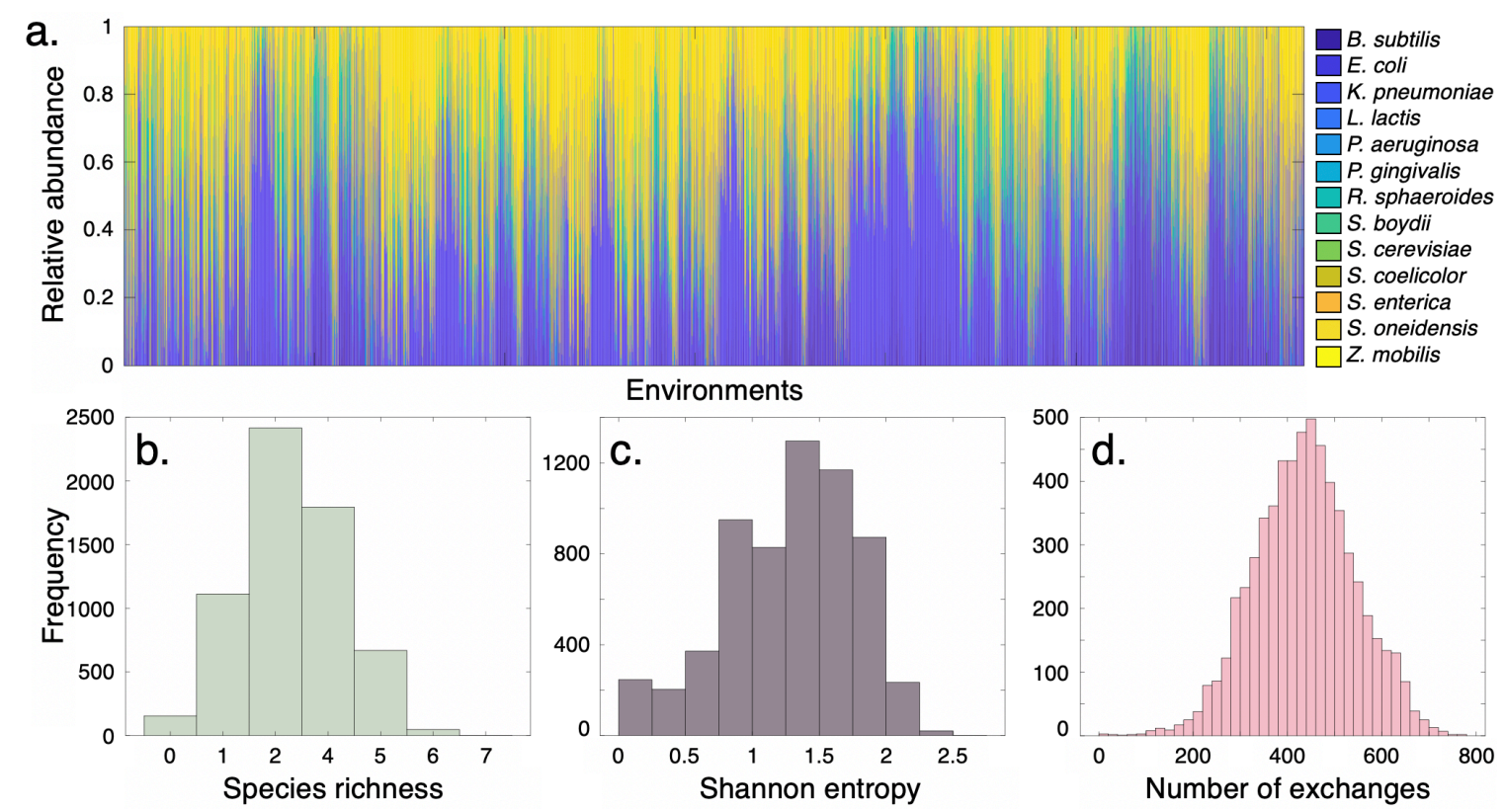

Figure 1. Structural and ecological properties of simulated 13-species communities. (a). Relative abundances of organisms after 24 hours of growth in all 6,196 combinatorial environmental compositions. (b-d). Distributions of species richness (b), Shannon entropy (c) and total number of exchanges (d) observed across all environments. Here, one exchange is defined as the transfer of a unique metabolite from one organism to another, e.g. the secretion of metabolite $m$ by organism $A$ and its absorption by organism $B$ represents one exchange. As our simulations contained 737 unique metabolites, the total possible number of exchanges (i.e. if each organism transfers each metabolite to each other organism) totals $\left(\begin{array}{c}13 \\ 2\end{array}\right) \times 737$, or 57,486 .

\section{A simple evolutionary algorithm rapidly identifies environmental compositions}

Having generated a wide array of phenotypic data, we designed a search algorithm to identify environments within our dataset that would result in specific community properties. This method, a genetic algorithm based on the process of natural selection [49-52], functions as follows: first, a population of $P$ environmental compositions is generated, each containing a random assortment of a maximum of $N$ unique nutrients. Community phenotypes (e.g. species abundances, interspecies interactions, metabolic secretions) on each environment are recorded, and each environment is scored according to the community function being optimized. A subset $\sigma$ consisting of the top-performing environments is then selected to be propagated to the next generation. The 
remaining $P-\sigma$ environments are generated by combining nutrients contained in the top $\sigma$ environments (crossover), and by introducing new nutrients (mutation) at rates defined by a parameter grid search (Supplementary Figure 1). These new $P$ environments are provided to the in silico communities, and the optimization process continues for $G$ generations (Figure 2). The objective of the algorithm is therefore to converge to a final set of environmental compositions that confer the desired properties on the community being tested, without prior knowledge of the dataset's structure.

We first applied this framework to identify environments that would maximize the final taxonomic balance of our communities. Though it is uncommon for organisms to be equally represented in natural settings [53-58], coexistence of multiple organisms is a desirable property for engineered consortia as it can enable tasks useful in biotechnology, such as metabolic division of labor [19,59]. As such, we sought to identify environments that resulted in relatively even species abundances. To do this, we applied the genetic algorithm to search for environments that would maximize the Shannon entropy of our in silico communities (see Methods). In order to gain a statistical representation of its performance, we ran our algorithm 50 separate times, each with different random initial compositions of $P=10$ environments. For each GA process, we recorded the generation at which the algorithm's proposed solutions crossed the $99^{\text {th }}$ percentile of all solutions as a way to quantify its performance. We found that, on average, our algorithm identified solutions that exceeded the $99^{\text {th }}$ percentile of Shannon entropy values after approximately 3 generations (Figure 3a, Supplementary Table 3). As each generation tested $P=10$ environments, this performance represents explicitly testing only 30 unique in silico experiments out of a total of 


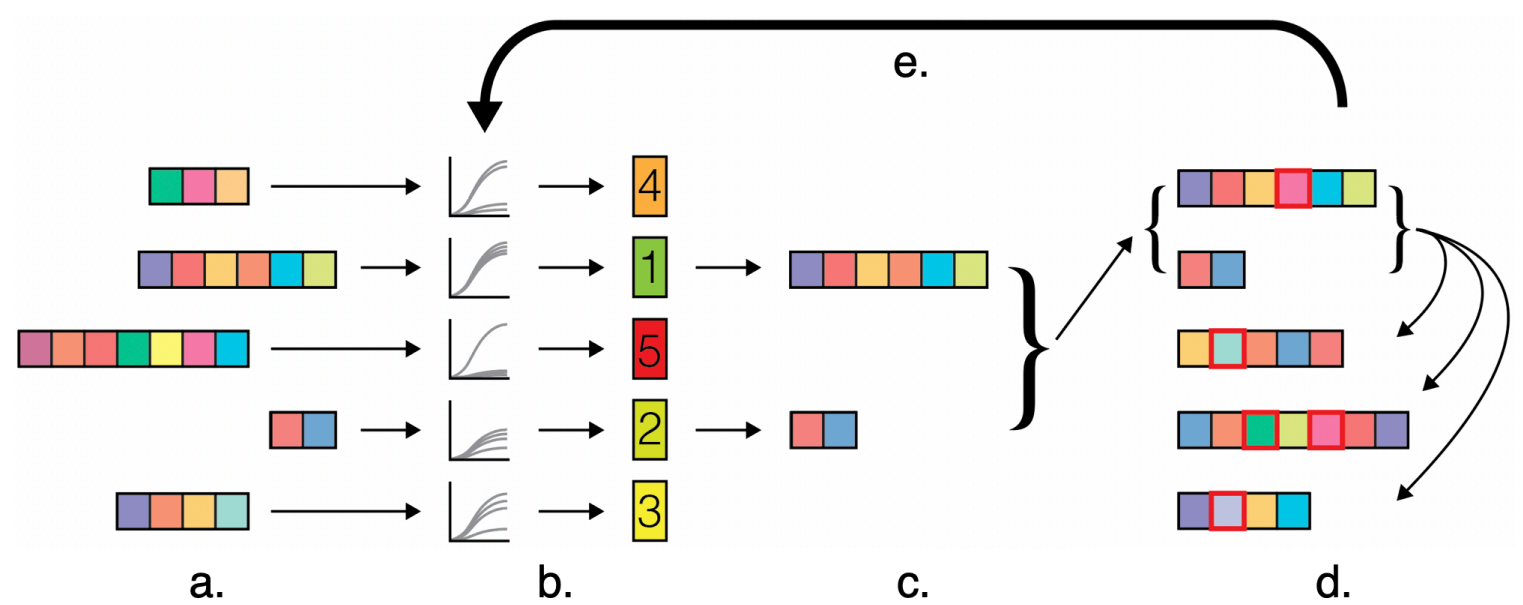

Figure 2. Schematic of genetic algorithm process for microbial community design. (a). A set of $P$ environmental compositions, each containing a varying number of limiting nutrients, is randomly generated. (b). The community phenotype observed in each environment is determined. As a representative example, this figure shows the GA process with taxonomic balance as the objective to be optimized. The environments are ranked according to their resulting communities' taxonomic balance, and (c) the top $\sigma$ environments are selected. Here, the environments that yielded the top $\sigma=2$ taxonomically balanced communities are chosen. (d). A new population of $P$ environments is generated. First, the top $\sigma$ environments are carried over into the new population as 'parents', and the remaining $P-\sigma$ 'offspring' environments are generated via multipoint crossover (i.e. the individual nutrients in the parents are shuffled to produce heterogeneous offspring). Variation is introduced into the new population via mutation, in which each individual element has a defined probability of being changed into a new one (red squares). (e). The process of environment ranking, propagation, crossover, and mutation is carried out for a total of $G$ generations.

6,196 total nutrient combinations. Though the algorithm generally converged quickly to nearoptimal solutions, we observed some variability in the specific environmental compositions it selected. For this particular objective, our method resulted in 12 distinct environmental compositions across the 50 different random seed environments, all of which showed high degrees of consistency and taxonomic balance in the resultant communities (Figure 3a, inset).

In addition to optimizing general ecological properties, we tested the capability of our algorithm to identify environments that would maximize more specific features. We first chose to optimize the relative abundances of individual organisms and selected B. subtilis, which grew in 2,130 out of 6,196 environments (Figure 1a), as a representative example. Again using 50 random seed sets, 
we found that the genetic algorithm was able to identify solutions that exceeded the $99^{\text {th }}$ percentile of B. subtilis abundances after approximately 8 generations on average (Figure 3b). We found that our algorithm converged to fewer distinct environmental compositions for this objective across our 50 random seeds, from which 4 distinct environments emerged (Figure 3b, inset). These environments nonetheless all conferred very high relative abundances to B. subtilis. Testing this capability for the remaining organisms revealed that similarly low numbers of generations were required to converge to optimal solutions (Supplementary Table 3), demonstrating the utility of this framework to identify environments that maximize individual species abundances.

Interspecies metabolic cooperation, often associated with microbial ecosystem stability, is a common target mechanism for community engineering [60,61]. Nonetheless, identifying environments that lead to the emergence of specific interactions remains an elusive task. We thus sought to determine whether our GA framework could also identify desired patterns of metabolic exchange from our computational dataset. We set the total number of interspecies exchanges as our objective function, in order to identify the environments that would maximize metabolic cooperation across all organisms. Our genetic algorithm was able to identify environments that surpassed the $99^{\text {th }}$ percentile of metabolic exchanges after 6 generations on average, representing a total of 60 in silico experiments (Figure 3c, Supplementary Table 3). Notably, the selected environments resulted in varied taxonomic compositions, ranging from those with high abundances of $E$. coli and $S$. oneidensis to those with more balanced compositions (Figure 3c, inset). This result suggests that the degree of metabolic exchange does not necessarily correlate with community taxonomic composition in our dataset, which parallels experimental observations showing conflicting correspondence between taxonomic structure and ecological function [62,63]. 

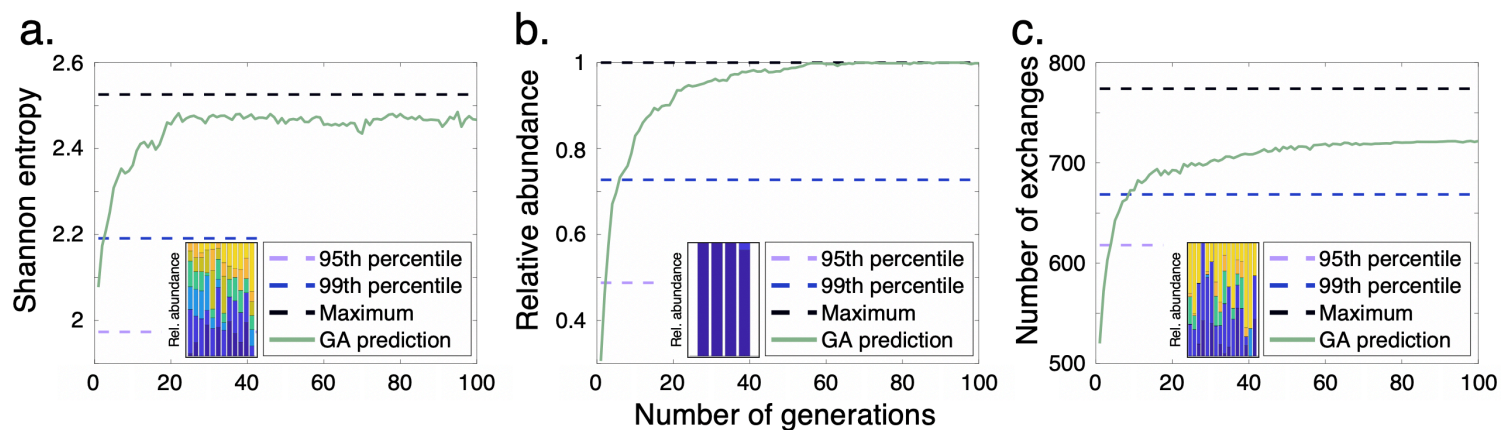

Figure 3. Performance of genetic algorithm on various ecological objectives. The average number of generations (using 50 random seed sets of $P=10$ environments) required to identify environments that surpassed the $99^{\text {th }}$ percentiles of (a) community Shannon entropy, (b) the relative abundance of $B$. subtilis, and (c) the total number of metabolic exchanges between organisms. Insets show the organism relative abundances of the top environmental conditions identified. All quantities, including results for optimization of the remaining 12 organisms' relative abundances, are found in Supplementary Table 3.

Given its ability to optimize the general prevalence of interspecies interactions, we also tested our algorithm on more specific patterns of secretion and exchange. In particular, we sought to determine if we could identify environments that resulted either in greater metabolic flux toward one particular organism or in greater overall secretion of a particular metabolite, as such specific phenomena are commonly leveraged for synthetic community design [60,61]. We again used $B$. subtilis as a representative organism to test the former capability, finding that our GA identified environments that surpassed the $99^{\text {th }}$ percentile of metabolic exchanges toward this organism after 9 generations on average. Testing the same capability with our remaining organisms as targets showed similar performance (Supplementary Table 3). We next set the net community-level output of specific metabolites from all organisms as an optimization target, in order to identify environments that would maximize their secretion. To do this, we selected 24 metabolites: 12 that were most highly secreted across all 6,196 simulations, and 12 that were least secreted. For the former set, we found that our algorithm identified solutions surpassing the $99^{\text {th }}$ percentile of secretion after, on average, 11 generations (Supplementary Table 3). However, our algorithm's 
performance suffered for metabolites with low secretion flux, requiring on average 143 generations to reach this same benchmark.

Despite eventually converging to near-optimal solutions for all of the metabolite secretion patterns we tested, the longer convergence time needed to identify solutions for some metabolites prompted us to quantify its dependence on the number of times a particular metabolite was observed to be secreted across all simulations. We thus analyzed the average number of generations needed to surpass the $99^{\text {th }}$ percentile for a given target metabolite with respect to the number of times it was observed in our dataset, finding that these two quantities were inversely proportional to each other (Supplementary Figure 2a). Though this effect reveals a limitation of our method (or indeed of FBA itself), a large number of generations is needed for a rare minority of objectives. For this dataset, we determined that the secretion of $61.4 \%$ of organic metabolites could be maximized within 50 generations, with only $21.5 \%$ of metabolites requiring over 100 generations (Supplementary Figure 2b).

\section{Searching for community phenotypes in larger combinatorial spaces}

Having benchmarked our GA framework on an exhaustive environment-phenotype mapping, we aimed to test its performance in a much larger search space. We thus applied it to determine whether certain environmental compositions could yield communities with highly specific organism relative abundances. This goal draws from efforts to precisely control organism ratios in mixed cultures, which is particularly relevant for synthetic communities applied to the synthesis of biofuels or chemicals [64-66]. Here, we sought to identify environments that would allow one of three organisms - B. subtilis, E. coli, and S. coelicolor - to reach a high abundance in a 
community (95\%), while maintaining the remaining two at low abundances $(2.5 \%$ each). We used a list of 154 limiting carbon sources from which we allowed our algorithm to select a maximum of 3. This search space, consisting of 596,904 unique environmental compositions, remains computationally expensive to test exhaustively using ecological modeling methods like dFBA and nearly impossible to test experimentally. Therefore, this application illustrates the capability of our GA framework to operate in an exploratory fashion within spaces that cannot be fully mapped.

To search this larger combinatorial space, we carried out dFBA simulations of our community in the selected environments as they were produced by the genetic algorithm, instead of generating a full environment-phenotype mapping a priori as above (see Methods). The quality of these environments was assessed by calculating the sum squared error (SSE) between the resulting community compositions and our target abundances $[0.95,0.025,0.025]$, and the objective of the GA was therefore to minimize this quantity. We found that, by iteratively searching this large combinatorial space, the GA framework successfully identified environments that allowed each organism to reach a high relative abundance while keeping the remaining two at low, but nonzero abundances (Figure 4a-c). Notably, the algorithm converged on multiple such environmental compositions, indicating a type of metabolic flexibility with regards to specific final taxonomic compositions.

We examined some of these environmental compositions in greater detail, identifying common interaction network structures that conferred the desired community phenotypes (Figure 4d-f). For example, in one of the environments that was selected to have B. subtilis dominate the community, 
a.

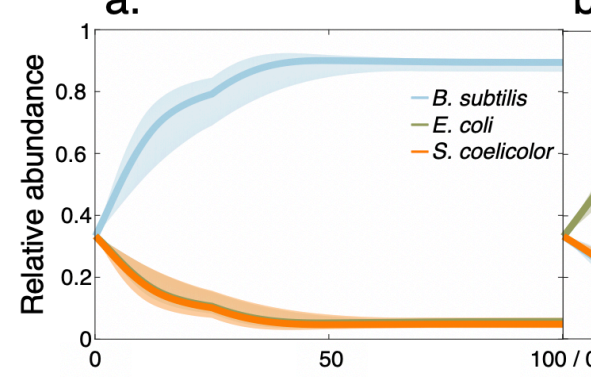

d.

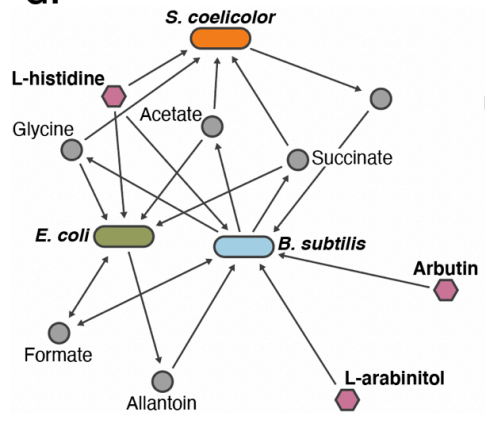

b.

C.

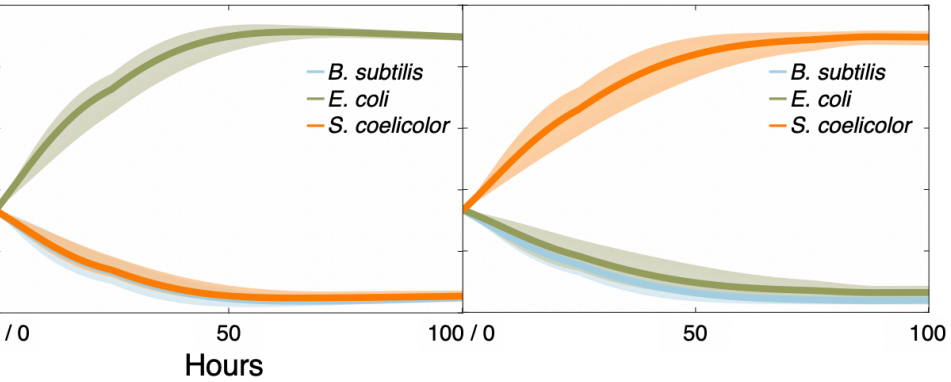

e.

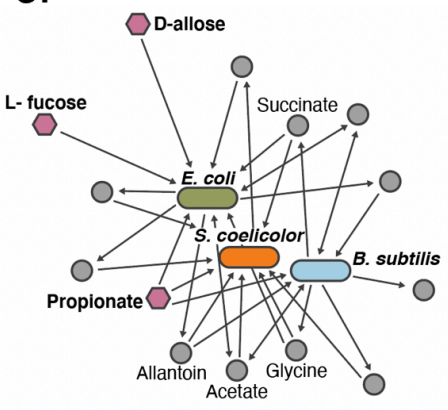

Figure 4. Simulated time-course trajectories of three-species community growth under various GAdetermined environments. Genetic algorithm was used to determine environments that would allow $B$. subtilis (a), E. coli (b), and $S$. coelicolor (c) to reach abundances of $95 \%$ while the remaining organisms remained in the communities at basal levels. Dark lines indicate mean growth curves and shaded regions encompass the maximum and minimum relative abundances for each organism across 10 random environment seed sets. $(d-f)$. Interaction network structures of representative environments that confer dominance to B. subtilis (d), E. coli (e), and S. coelicolor (f). Elongated ovals represent organisms, pink hexagons represent primary nutrients (environmental composition), and gray circles represent exchanged metabolites. Select commonly-exchanged metabolites are labeled.

our dFBA simulation revealed that it was the exclusive consumer of two out of three primary nutrients, while the third nutrient was shared between the three organisms (Figure 4d). A similar structure was also observed for the environments that optimized dominance of $E$. coli and $S$. coelicolor (Figure 4e, f), suggesting that nutrient specificity was a major driving force of organism dominance in these communities. We also observed dense networks of metabolic byproduct exchange, with molecules such as acetate, formate, glycine, and succinate being frequently transferred between organisms, paralleling previous experimental observations of organic acid transfer [67-69]. Given that a crucial element of our objective was for two organisms to persist at 
low abundances, these metabolic exchanges (along with consumption of a third primary nutrient) may be allowing the communities to remain stable with the desired taxonomic proportions.

\section{Discussion}

The rational design of multispecies communities toward defined phenotypes is a challenging, yet enticing, goal of synthetic ecology. As the phenotypic traits of communities in complex settings remain difficult to predict [28,70,71], fulfilling this potential will require a synthesis of computational and experimental methods that focus on different aspects of these communities [10,72-74]. Here, we used in silico microbial communities to show how their ecological properties can be modulated via environmental modification, and presented a search algorithm to identify specific nutrient combinations that would result in desired phenotypes. We showed how this algorithm was quickly able to identify high-quality solutions for a variety of ecological objectives: from overall taxonomic balance to specific organism abundances and patterns of metabolic secretion and exchange. Given these capabilities, this method represents a computationallyinexpensive way to rapidly screen very large combinatorial spaces to produce desired community properties. Moreover, in addition to optimizing the various objectives tested here, our dFBAgenetic algorithm framework can be extended to encompass a greater number of important environmental attributes, such as varying nutrient concentrations and spatiotemporal nutrient variation $[41,42]$.

Despite the flexibility and mechanistic insight afforded by a dFBA approach, engineering synthetic ecosystems in vitro will inevitably require experimental validation of modeling predictions. Our approach can be applied to this goal in two ways. First, in silico analogs of a desired experimental 
system may be iteratively screened as we have performed here, and the final environments generated by the genetic algorithm may then be explicitly tested experimentally. In this way, our method serves to generate an accessible number of testable hypotheses pertaining to specific ecological systems. Pairing of flux balance models and confirmatory experiments in this way has been used extensively to obtain a greater understanding of organism function as well as exploring previously unknown phenotypes [32,75-78]. However, as high-quality genome-scale reconstructions are limited to relatively few well-characterized model organisms, the applicability of this method is limited to a small set of community taxonomic compositions. A second strategy can forgo the dFBA component altogether, and use the evolutionary algorithm as a way to search through experimentally-derived community phenotypic data. As we showed how the GA was able to reach high-quality solutions with relatively few experimental data points, one could envision implementing a similar framework alongside the in vitro testing of a community. Here, iterative cycles of testing could be fed into a GA structure, which could inform the next stage of experiments. Given the increasing accessibility of high-throughput platforms for microbial ecology (e.g. microfluidics, microdroplets, etc.) [37-39], a search algorithm like ours can feasibly be deployed alongside such techniques to rapidly reach predefined community objectives.

\section{Methods}

\section{Generation of environment-phenotype mapping with $\mathrm{dFBA}$}

We employed a dynamic flux balance analysis (dFBA) method [40] to test the response of a multispecies community in a combinatorial assortment of environments. This process, which was carried out using the COMETS (Computation of Microbial Ecosystems in Time and Space) software package $[41,42]$, allowed us to extract a wide array of phenotypic data from simulated 
microbial communities. The process by which COMETS carries out these simulations has been outlined in detail in previous publications $[33,41,42]$, and was carried out in the following way for our application: (1) Combinatorial environments were generated by combining an in silico minimal medium with limiting quantities of a set of carbon sources. This minimal medium, modeled after the composition of M9, contained molecules necessary for growth such as water, ions, and sources of nitrogen, phosphorus, and sulfur introduced at nonlimiting concentrations. Limiting amounts of 20 carbon sources were then added on an environment-by environment basis. These nutrients, an assortment of sugars, organic acids, and amino acids (Supplementary Table 2), were added in all combinations of up to 4 at equimolar ratios such that the total concentration of carbon in each environment was $50 \mathrm{mM} \mathrm{C}$ in $400 \mu \mathrm{L}$. This scheme resulted in 6,196 unique environmental compositions. (2) Genome-scale reconstructions [31,32] of 13 specific microbial organisms were placed in our in silico media compositions. These organism-specific models span a wide range of taxa and metabolic strategies, and were selected to maximize variation in endpoint community composition and interactions across our combinatorial environments (Supplementary Table 1). Based on an approximate total inoculum of OD600 0.05 corresponding to $1.6 \times 10^{7}$ cells in $400 \mu \mathrm{L}$, and a cell mass of $2.8 \times 10^{-13}$ grams dry weight $(\mathrm{gDW})$ [79], all 13 organisms were inoculated into our in silico media at equal ratios of $3.45 \times 10^{-7} \mathrm{gDW}$ for a total inoculum of $4.48 \times 10^{-6} \mathrm{gDW}$ (OD600 0.05 total). The growth of these mixed cultures was then simulated in COMETS over the course of 24 hours, with a death rate parameter of 0.1 and a timestep of 0.01 hours [41]. Once completed, the total final biomass quantities, relative abundances, and secreted and absorbed metabolites for each environment were recorded. 
For our second, exploratory application of the genetic algorithm, a larger pool of 154 carbon sources was used from which a maximum of three nutrients were selected per environment, resulting in 596,904 unique environmental compositions. Three organism genome-scale reconstructions (B. subtilis, E. coli, and S. coelicolor (Supplementary Table 1)) from our list of 13 were used and inoculated into our environments in a similar way to that described above. However, we did not explicitly simulate the community phenotypes in all combinatorial environments. Instead, only the environmental compositions selected by the genetic algorithm in each generation were tested and their performance recorded as above.

\section{Design and parametrization of genetic algorithm}

A genetic algorithm is a search heuristic based on the principle of evolution by natural selection, which optimizes a particular objective function via the modification of a population of individual solutions [50]. Our selection of a genetic algorithm was based on its applicability to the optimization of nonlinear problems, which reflect the nature of complex environment-phenotype relationships in microbial communities. In our implementation, the individual solutions being modified are unique environmental compositions expressed as vectors denoting the presence of a particular nutrient. The objective function varied according to the phenotype being optimized. In this work, we selected a number of different objective functions to maximize, namely: (1) the overall Shannon entropy of a community as a reflection of taxonomic balance, (2) the relative abundances of each of the 13 in silico organisms, (3) the total number of metabolic exchanges, (4) the total metabolic flux directed at each of the 13 in silico organisms, (5) the total secretion flux of 24 different metabolic byproducts, and (6) the approximation of target relative abundances. The modifications of different solutions take place over the course of multiple 'generations,' in which 
each solution is scored according to the phenotype being optimized, and the best solutions are used to seed a new generation of candidate solutions. This process continues with the intent of converging on a set of optimal solutions.

Our implementation of the GA begins with a randomly-generated population made up of $P$ environmental compositions. In order to demonstrate its extensibility to be used in parallel to an in vitro experimental system, we sought to minimize the number of environmental compositions $P$ tested in each generation. Therefore, we limited the number of compositions to an experimentally-tractable $P=10$ in each generation. The $P$ environments were initialized with random assortments of up to $N$ nutrients $(N=4$ for our initial benchmarking study, and $N=3$ for the second exploratory example). The community phenotypes resulting from each environment in the population (either pre-generated dFBA data in our benchmarking study, dFBA data generated as-needed in our exploratory example, and, in principle, experimental data if being used alongside an in vitro system) are recorded and used to rank each environment according to the objective function. The algorithm then selects the top $\sigma$ environments to serve as 'parents' to the next generation of solutions. Having selected a set of $\sigma$ parent environments, the algorithm then uses them to populate a new generation of $P$ candidate solutions. This step takes place through processes of crossover (the individual elements of the parents are combined) and mutation (random, new elements are introduced). In our implementation, the parent nutrient vectors are linearized, and the remaining $P-\sigma$ environments are populated with random assortments of the nutrients contained in the parent vector. Mutation then occurs, in which the individual nutrients of all $P$ environments are subject to being randomly replaced by a nutrient yet unused in the set. The number of environments subject to crossover, as well as the probability of any individual nutrient 
being subject to mutation, are defined by crossover and mutation probabilities $p_{C}$ and $p_{M}$, respectively.

We determined optimal values for the crossover and mutation probabilities $p_{C}$ and $p_{M}$ via a parameter grid search. To do this, we selected three representative objective functions: (1) maximization of community Shannon entropy, (2) maximization of the relative abundance of $B$. subtilis, and (3) maximization of the total number of metabolic exchanges. We then varied the values of $p_{C}$ from 0 to 1 in intervals of 0.1 , and the values of $p_{M}$ from 0 to 0.45 in intervals of 0.05 . The values of $p_{M}$ were maintained under 0.5 in order to ensure the GA process would not diverge from optimal solutions via excessive mutation. For each pairing of $p_{C}$ and $p_{M}$, we applied our GA 50 times, each with a random seed set of $P=10$ different environments. We then evaluated the performance of the GA for each objective using a performance score $S$. This score is based on a combination of two metrics: (1) the number of generations required for a set of solutions to surpass the $99^{\text {th }}$ percentile of a given objective $\left(G_{99}\right)$ and $(2)$ the percentile reached at the final generation of the algorithm $P r_{\text {end }}$. Since a lower $G_{99}$ denotes better performance, the performance score $S$ is defined as follows:

$$
S=\left(1-\widetilde{G_{99}}\right)+\left(\widetilde{P r_{e n d}}\right)
$$

Where $\widetilde{G_{99}}$ and $\widetilde{P r_{\text {end }}}$ are normalized from 0 to 1 , such that $S$ can range from 0 to 2 . We found that the best $\left[p_{C}, p_{M}\right]$ values were $[0.7,0.45]$ for our first objective, $[1,0.3]$ for our second, and $[0.8$, 0.45] for our third (Supplementary Figure 2). Interestingly, while our $p_{C}$ values were consistent with commonly-used crossover parameter values [80], we noticed low variability in performance scores $S$ with respect to changing mutation probabilities $p_{M}$. We thus used an average best $\left[p_{C}, p_{M}\right]$ values $([0.8,0.4])$ for all of our GA objectives. For a population size of $P=10$, a $p_{C}$ value of 0.8 
therefore results in $\sigma=2$ environments being selected to carry over and seed a new generation.

Once a new generation of environments is created via carryover, crossover, and mutation, the community phenotype in in each environment is recorded, and the environments are ranked. In our implementation, this process of selection continues until a maximum of generations $G$ is reached (Figure 2).

\section{Data accessibility}

MATLAB scripts for running the genetic algorithm are available at github.com/segrelab/EvolutionaryAlgorithms.

\section{Authors' contributions}

A.R.P and D.S. designed the research. A.R.P. developed the algorithm framework, collected data, and designed and performed simulations. A.R.P. and D.S. wrote the manuscript. Both authors read and approved the final manuscript.

\section{Competing interests}

The authors declare that no competing interests exist in relation to this manuscript.

\section{Funding}

A.R.P. is supported by a Howard Hughes Medical Institute Gilliam Fellowship and a National Academies of Sciences, Engineering, and Medicine Ford Foundation Predoctoral Fellowship. We gratefully acknowledge support from the U.S. Department of Energy, Office of Science, Office of Biological \& Environmental Research through the Microbial Community Analysis and Functional 
Evaluation in Soils SFA Program (m-CAFEs) under contract number DE-AC02-05CH11231 to

Lawrence Berkeley National Laboratory, as well as the National Institutes of Health (grants 5R01DE024468, R01GM121950), the National Science Foundation (grants 1457695 and NSFOCE-BSF 1635070), the Human Frontiers Science Program (grant RGP0020/2016), and the Boston University Interdisciplinary Biomedical Research Office.

\section{Acknowledgements}

The authors wish to thank members of the Segrè lab for inspiring conversations. We are especially grateful to Joshua Goldford, Mark Kon, and Dileep Kishore for helpful discussions, and to David Bernstein, Melisa Osborne, and Devlin Moyer for their constructive comments on the manuscript.

\section{References}

1. Venter JC et al. 2004 Environmental genome shotgun sequencing of the Sargasso Sea. Science 304, 66-74. (doi:10.1126/science.1093857)

2. Sogin ML, Morrison HG, Huber JA, Mark Welch D, Huse SM, Neal PR, Arrieta JM, Herndl GJ. 2006 Microbial diversity in the deep sea and the underexplored 'rare biosphere'. Proceedings of the National Academy of Sciences of the United States of America 103, 12115-20. (doi:10.1073/pnas.0605127103)

3. Tecon R, Or D. 2017 Biophysical processes supporting the diversity of microbial life in soil. FEMS Microbiology Reviews 41, 599-623. (doi:10.1093/femsre/fux039)

4. The Human Microbiome Project Consortium. 2012 Structure, function and diversity of the healthy human microbiome. Nature 486, 207-214. (doi:10.1038/nature11234)

5. Falkowski PG, Fenchel T, Delong EF. 2008 The Microbial Engines That Drive Earth's Biogeochemical Cycles. Science 320, 1034-1039. (doi:10.1126/science.1153213)

6. Sunagawa S et al. 2015 Structure and function of the global ocean microbiome. Science 348, 1261359. (doi:10.1126/science.1261359)

7. Gilbert JA, Jansson JK, Knight R. 2014 The Earth Microbiome project: successes and aspirations. BMC Biology 12, 69. (doi:10.1186/s12915-014-0069-1)

8. Turnbaugh PJ, Ley RE, Hamady M, Fraser-Liggett CM, Knight R, Gordon JI. 2007 The Human Microbiome Project. Nature 449, 804-810. (doi:10.1038/nature06244)

9. Teague BP, Weiss R. 2015 Synthetic communities, the sum of parts. Science 349.

10. Zomorrodi AR, Segrè D. 2016 Synthetic Ecology of Microbes: Mathematical Models and Applications. Journal of Molecular Biology 428, 837-861. (doi:10.1016/j.jmb.2015.10.019)

11. Vrancken G, Gregory AC, Huys GRB, Faust K, Raes J. 2019 Synthetic ecology of the human gut microbiota. Nature Reviews Microbiology (doi:10.1038/s41579-019-0264-8)

12. Kang D, Jacquiod S, Herschend J, Wei S, Nesme J, Sørensen SJ. 2020 Construction of Simplified 
Microbial Consortia to Degrade Recalcitrant Materials Based on Enrichment and Dilution-toExtinction Cultures. Frontiers in Microbiology 10. (doi:10.3389/fmicb.2019.03010)

13. Mahajan N, Gupta P. 2015 New insights into the microbial degradation of polyurethanes. RSC Advances 5, 41839-41854. (doi:10.1039/C5RA04589D)

14. Minty JJ, Singer ME, Scholz SA, Bae C-H, Ahn J-H, Foster CE, Liao JC, Lin XN. 2013 Design and characterization of synthetic fungal-bacterial consortia for direct production of isobutanol from cellulosic biomass. Proceedings of the National Academy of Sciences of the United States of America 110, 14592-14597. (doi:10.1073/pnas.1218447110)

15. McCarty NS, Ledesma-Amaro R. 2019 Synthetic Biology Tools to Engineer Microbial Communities for Biotechnology. Trends in Biotechnology 37, 181-197. (doi:10.1016/j.tibtech.2018.11.002)

16. Jones JA et al. 2017 Complete Biosynthesis of Anthocyanins Using E. coli Polycultures. mBio 8. (doi:10.1128/mBio.00621-17)

17. Zhang H, Pereira B, Li Z, Stephanopoulos G. 2015 Engineering Escherichia coli coculture systems for the production of biochemical products. Proceedings of the National Academy of Sciences of the United States of America 112, 8266-8271. (doi:10.1073/pnas.1506781112)

18. Zhou K, Qiao K, Edgar S, Stephanopoulos G. 2015 Distributing a metabolic pathway among a microbial consortium enhances production of natural products. Nature Biotechnology 33, 377-383. (doi:10.1038/nbt.3095)

19. Ziesack M et al. 2019 Engineered Interspecies Amino Acid Cross-Feeding Increases Population Evenness in a Synthetic Bacterial Consortium. mSystems 4. (doi:10.1128/mSystems.00352-19)

20. Konopka A, Lindemann S, Fredrickson J. 2015 Dynamics in microbial communities: unraveling mechanisms to identify principles. The ISME Journal 9, 1488-1495. (doi:10.1038/ismej.2014.251)

21. Lindemann SR, Bernstein HC, Song H-S, Fredrickson JK, Fields MW, Shou W, Johnson DR, Beliaev AS. 2016 Engineering microbial consortia for controllable outputs. The ISME Journal 10, 2077-2084. (doi:10.1038/ismej.2016.26)

22. Smits SA et al. 2017 Seasonal cycling in the gut microbiome of the Hadza hunter-gatherers of Tanzania. Science 357, 802-806. (doi:10.1126/science.aan4834)

23. Yang Q, Liang Q, Balakrishnan B, Belobrajdic DP, Feng Q-J, Zhang W. 2020 Role of Dietary Nutrients in the Modulation of Gut Microbiota: A Narrative Review. Nutrients 12, 381. (doi:10.3390/nu12020381)

24. Fonte ES, Amado AM, Meirelles-Pereira F, Esteves FA, Rosado AS, Farjalla VF. 2013 The Combination of Different Carbon Sources Enhances Bacterial Growth Efficiency in Aquatic Ecosystems. Microbial Ecology 66, 871-878. (doi:10.1007/s00248-013-0277-1)

25. Preusser S, Marhan S, Poll C, Kandeler E. 2017 Microbial community response to changes in substrate availability and habitat conditions in a reciprocal subsoil transfer experiment. Soil Biology and Biochemistry 105, 138-152. (doi:10.1016/j.soilbio.2016.11.021)

26. Replansky T, Bell G. 2009 The relationship between environmental complexity, species diversity and productivity in a natural reconstructed yeast community. Oikos 118, 233-239. (doi:10.1111/j.1600-0706.2008.16948.x)

27. Pacheco AR, Moel M, Segrè D, Segre D. 2018 Costless metabolic secretions as drivers of interspecies interactions in microbial ecosystems. bioRxiv, 1-42. (doi:10.1101/300046)

28. Pacheco AR, Segrè D. 2020 The effects of environmental complexity on microbial community yield and structure. bioRxiv (doi:https://doi.org/10.1101/2020.07.09.195404)

29. Muscarella ME, Boot CM, Broeckling CD, Lennon JT. 2019 Resource heterogeneity structures aquatic bacterial communities. ISME Journal 13, 2183-2195. (doi:10.1038/s41396-019-0427-7)

30. Estrela S, Sanchez-Gorostiaga A, Vila JCC, Sanchez A. 2020 Nutrient dominance governs the assembly of microbial communities in mixed nutrient environments. bioRxiv , 2020.08.06.239897. (doi:10.1101/2020.08.06.239897)

31. Orth JD, Thiele I, Palsson BØO. 2010 What is flux balance analysis? Nature Biotechnology 28, 245-248. (doi:10.1038/nbt.1614) 
32. Bordbar A, Monk JM, King ZA, Palsson BO. 2014 Constraint-based models predict metabolic and associated cellular functions. Nature Reviews Genetics 15, 107-120. (doi:10.1038/nrg3643)

33. Pacheco AR, Moel M, Segrè D. 2019 Costless metabolic secretions as drivers of interspecies interactions in microbial ecosystems. Nature Communications 10, 103. (doi:10.1038/s41467-01807946-9)

34. Swenson W, Wilson DS, Elias R. 2000 Artificial ecosystem selection. Proceedings of the National Academy of Sciences of the United States of America 97, 9110-9114. (doi:10.1073/pnas.150237597)

35. Arias-Sánchez FI, Vessman B, Mitri S. 2019 Artificially selecting microbial communities: If we can breed dogs, why not microbiomes? PLoS Biology 17, 1-8. (doi:10.1371/journal.pbio.3000356)

36. Chang C, Osborne ML, Bajic D, Sanchez A. 2020 Artificially selecting microbial communities using propagule strategies. bioRxiv , 1-19.

37. Cira NJ, Ho JY, Dueck ME, Weibel DB. 2012 A self-loading microfluidic device for determining the minimum inhibitory concentration of antibiotics. Lab Chip 12, 1052-1059. (doi:10.1039/C2LC20887C)

38. Kaminski TS, Scheler O, Garstecki P. 2016 Droplet microfluidics for microbiology: techniques, applications and challenges. Lab on a Chip 16, 2168-2187. (doi:10.1039/C6LC00367B)

39. Kehe J et al. 2019 Massively parallel screening of synthetic microbial communities. Proceedings of the National Academy of Sciences of the United States of America 116, 12804-12809. (doi:10.1073/pnas.1900102116)

40. Mahadevan R, Edwards JS, Doyle FJ. 2002 Dynamic Flux Balance Analysis of Diauxic Growth in Escherichia coli. Biophysical Journal 83, 1331-1340. (doi:10.1016/S0006-3495(02)73903-9)

41. Harcombe WR et al. 2014 Metabolic resource allocation in individual microbes determines ecosystem interactions and spatial dynamics. Cell Reports 7, 1104-1115. (doi:10.1016/j.celrep.2014.03.070)

42. Dukovski I et al. 2020 Computation Of Microbial Ecosystems in Time and Space (COMETS): An open source collaborative platform for modeling ecosystems metabolism. arXiv

43. Henson MA, Hanly TJ. 2014 Dynamic flux balance analysis for synthetic microbial communities. IET systems biology 8, 214-29. (doi:10.1049/iet-syb.2013.0021)

44. Magnúsdóttir S et al. 2016 Generation of genome-scale metabolic reconstructions for 773 members of the human gut microbiota. Nature Biotechnology (doi:10.1038/nbt.3703)

45. Flahaut NAL, Wiersma A, Van De Bunt B, Martens DE, Schaap PJ, Sijtsma L, Dos Santos VAM, De Vos WM. 2013 Genome-scale metabolic model for Lactococcus lactis MG1363 and its application to the analysis of flavor formation. Applied Microbiology and Biotechnology 97, 87298739. (doi:10.1007/s00253-013-5140-2)

46. Mazumdar V, Snitkin ES, Amar S, Segrè D. 2009 Metabolic network model of a human oral pathogen. Journal of Bacteriology 91, 74-90. (doi:10.1128/JB.01123-08)

47. Imam S, Yilmaz S, Sohmen U, Gorzalski AS, Reed JL, Noguera DR, Donohue TJ. 2011 IRsp1095: A genome-scale reconstruction of the Rhodobacter sphaeroides metabolic network. BMC Systems Biology 5, 116. (doi:10.1186/1752-0509-5-116)

48. Motamedian E, Saeidi M, Shojaosadati SA. 2016 Reconstruction of a charge balanced genome-scale metabolic model to study the energy-uncoupled growth of Zymomonas mobilis ZM1. Molecular BioSystems 12, 1241-1249. (doi:10.1039/C5MB00588D)

49. Mitchell M. 1996 An Introduction to Genetic Algorithms. MIT Press.

50. Holland JH. 1975 Adaptation in Natural and Artificial Systems. Ann Arbor, MI: University of Michigan Press.

51. Dawn Thompson J. 20163 - Statistical Alignment Approaches. In Statistics for Bioinformatics (ed J Dawn Thompson), pp. 43-51. Oxford: Elsevier. (doi:https://doi.org/10.1016/B978-1-78548-2168.50004-X)

52. Hamblin S. 2013 On the practical usage of genetic algorithms in ecology and evolution. Methods in Ecology and Evolution 4, 184-194. (doi:10.1111/2041-210X.12000)

53. Eguíluz VM, Salazar G, Fernández-Gracia J, Pearman JK, Gasol JM, Acinas SG, Sunagawa S, 
Irigoien X, Duarte CM. 2019 Scaling of species distribution explains the vast potential marine prokaryote diversity. Scientific Reports 9, 18710. (doi:10.1038/s41598-019-54936-y)

54. Locey KJ, Lennon JT. 2016 Scaling laws predict global microbial diversity. Proceedings of the National Academy of Sciences of the United States of America 113, 5970-5. (doi:10.1073/pnas.1521291113)

55. Hoffmann KH, Rodriguez-Brito B, Breitbart M, Bangor D, Angly F, Felts B, Nulton J, Rohwer F, Salamon P. 2007 Power law rank-abundance models for marine phage communities. FEMS Microbiology Letters 273, 224-8. (doi:10.1111/j.1574-6968.2007.00790.x)

56. Acinas SG, Klepac-Ceraj V, Hunt DE, Pharino C, Ceraj I, Distel DL, Polz MF. 2004 Fine-scale phylogenetic architecture of a complex bacterial community. Nature 430, 551-4. (doi:10.1038/nature02649)

57. Hughes JB, Hellmann JJ, Ricketts TH, Bohannan BJ. 2001 Counting the uncountable: statistical approaches to estimating microbial diversity. Applied and Environmental Microbiology 67, 4399406. (doi:10.1128/aem.67.10.4399-4406.2001)

58. Schloss PD, Handelsman J. 2006 Toward a Census of Bacteria in Soil. PLoS Computational Biology 2, e92. (doi:10.1371/journal.pcbi.0020092)

59. Alnahhas RN, Sadeghpour M, Chen Y, Frey AA, Ott W, Josić K, Bennett MR. 2020 Majority sensing in synthetic microbial consortia. Nature Communications 11, 3659. (doi:10.1038/s41467020-17475-z)

60. Cavaliere M, Feng S, Soyer OS, Jiménez JI. 2017 Cooperation in microbial communities and their biotechnological applications. Environmental Microbiology 19, 2949-2963. (doi:10.1111/14622920.13767)

61. Hays SG, Patrick WG, Ziesack M, Oxman N, Silver PA. 2015 Better together: engineering and application of microbial symbioses. Current Opinion in Biotechnology 36, 40-49. (doi:10.1016/J.COPBIO.2015.08.008)

62. Louca S, Jacques SMS, Pires APF, Leal JS, Srivastava DS, Parfrey LW, Farjalla VF, Doebeli M. 2017 High taxonomic variability despite stable functional structure across microbial communities. Nature Ecology \& Evolution 1, 0015. (doi:10.1038/s41559-016-0015)

63. Bao Y, Guo Z, Chen R, Wu M, Li Z, Lin X, Feng Y. 2020 Functional community composition has less environmental variability than taxonomic composition in straw-degrading bacteria. Biology and Fertility of Soils 56, 869-874. (doi:10.1007/s00374-020-01455-y)

64. Tsai S-L, Goyal G, Chen W. 2010 Surface Display of a Functional Minicellulosome by Intracellular Complementation Using a Synthetic Yeast Consortium and Its Application to Cellulose Hydrolysis and Ethanol Production. Applied and Environmental Microbiology 76, 7514-7520. (doi:10.1128/AEM.01777-10)

65. Zhang H, Stephanopoulos G. 2016 Co-culture engineering for microbial biosynthesis of 3-aminobenzoic acid in Escherichia coli. Biotechnology Journal 11, 981-987. (doi:10.1002/biot.201600013)

66. Stephens K, Pozo M, Tsao C-Y, Hauk P, Bentley WE. 2019 Bacterial co-culture with cell signaling translator and growth controller modules for autonomously regulated culture composition. Nature Communications 10, 4129. (doi:10.1038/s41467-019-12027-6)

67. LaSarre B, McCully AL, Lennon JT, McKinlay JB. 2017 Microbial mutualism dynamics governed by dose-dependent toxicity of cross-fed nutrients. The ISME Journal 11, 337-348. (doi:10.1038/ismej.2016.141)

68. Sousa DZ, Smidt H, Alves MM, Stams AJM. 2009 Ecophysiology of syntrophic communities that degrade saturated and unsaturated long-chain fatty acids. FEMS Microbiology Ecology 68, 257272. (doi:10.1111/j.1574-6941.2009.00680.x)

69. Lee IH, Fredrickson AG, Tsuchiya HM. 1976 Dynamics of mixed cultures ofLactobacillus plantarum andPropionibacterium shermanii. Biotechnology and Bioengineering 18, 513-526. (doi:10.1002/bit.260180406)

70. Momeni B, Xie L, Shou W. 2017 Lotka-Volterra pairwise modeling fails to capture diverse pairwise microbial interactions. eLife 6, 1-34. (doi:10.7554/eLife.25051.001) 
71. Clark RL, Connors BM, Stevenson DM, Hromada SE, Hamilton JJ, Amador-Noguez D, Venturelli OS. 2020 Design of synthetic human gut microbiome assembly and function. bioRxiv , 1-30. (doi:https://doi.org/10.1101/2020.08.19.241315)

72. Widder S et al. 2016 Challenges in microbial ecology: building predictive understanding of community function and dynamics. The ISME Journal 10, 2557-2568. (doi:10.1038/ismej.2016.45)

73. Escalante AE, Rebolleda-Gómez M, Benítez M, Travisano M. 2015 Ecological perspectives on synthetic biology: insights from microbial population biology. Frontiers in Microbiology 6, 143. (doi:10.3389/fmicb.2015.00143)

74. Said S Ben, Or D. 2017 Synthetic microbial ecology: Engineering habitats for modular consortia. Frontiers in Microbiology 8. (doi:10.3389/fmicb.2017.01125)

75. Lewis NE et al. 2010 Large-scale in silico modeling of metabolic interactions between cell types in the human brain. Nature Biotechnology 28, 1279-1285. (doi:10.1038/nbt.1711)

76. Oberhardt MA, Palsson BO, Papin JA. 2009 Applications of genome-scale metabolic reconstructionS. Molecular Systems Biology 5. (doi:10.1038/msb.2009.77)

77. Becker SA, Feist AM, Mo ML, Hannum G, Palsson BO, Herrgard MJ. 2007 Quantitative prediction of cellular metabolism with constraint-based models: the COBRA Toolbox. Nature Protocols 2. (doi:10.1038/nprot.2007.99)

78. Maoz BM et al. 2018 A linked organ-on-chip model of the human neurovascular unit reveals the metabolic coupling of endothelial and neuronal cells. Nature Biotechnology 36, 865-874. (doi:10.1038/nbt.4226)

79. Neidhardt FC, Ingraham JL, Schaechter M. 1990 Physiology of the Bacterial Cell. Sinauer Associates Inc.

80. Hassanat A, Almohammadi K, Alkafaween E, Abunawas E, Hammouri A, Prasath VBS. 2019 Choosing Mutation and Crossover Ratios for Genetic Algorithms-A Review with a New Dynamic Approach. Information 10, 390. (doi:10.3390/info10120390) 